\title{
CP VIOLATION IN THE LEPTON SECTOR WITH SMALL NEUTRINO MASSES
}

\author{
J.-M. FRÈRE ${ }^{1 *}$ and Jiang LIU \\ Randall Laboratory of Physics, University of Michigan, Ann Arbor, MI 48109, USA \\ Received 14 February 1989 \\ (Revised 3 April 1989)
}

\begin{abstract}
Small, even vanishing neutrino masses are consistent with large $C P$ violation effects in the leptonic sector. As an example, and a proof of existence of this situation, we present a model based on the $S U(2)_{L} \times S U(2)_{R} \times U(1)$ gauge symmetry, where the fermion content has been extended to include a gauge singlet lepton $S_{\mathrm{L}}$. Interesting features of this model (which should be seen as a mere example) are: (1) neutrino masses can either vanish or be in the range where the Mikheyev-Smirnov-Wolfenstein (MSW) mechanism for the solar neutrino puzzle is operative, and (2) $C P$ violation effects in the lepton sector are manifestly large and therefore accessible to current experiments. In particular, it is found that the electron electric dipole moment, $d_{\mathrm{e}}$, can be of order $10^{-24} e \cdot \mathrm{cm}$; if generation mixing is negligible, the $\mu$ longitudinal polarization, $P_{\mathrm{L}}$, in the decay $\mathrm{K}_{\mathrm{L}} \rightarrow \mu \bar{\mu}$ can be in the range $10^{-1}-10^{-3}$.
\end{abstract}

\section{Introduction}

Our present understanding of the neutrino mass is still far from clear [1]. While experiments are so far consistent with massless neutrinos, there is no compelling reason for this to remain true. (In particular, there is no gauge principle which forces $m_{\nu}=0$.) During the last few years, considerable attention has been focused on the possibility of using the Mikheyev-Smirnov-Wolfenstein (MSW) mechanism [2] to understand the solar neutrino puzzle [3]. For such a mechanism, which results from a matter-enhanced conversion between two different types of neutrinos, to be effective, the difference of squared masses of the neutrinos must be within the range [4]

$$
10^{-7} \mathrm{eV}^{2} \leq \Delta m^{2} \leq 10^{-4} \mathrm{eV}^{2},
$$

and the mixing between these two species must obey

$$
\sin ^{2} 2 \theta \geq 10^{-3} \text {. }
$$

${ }^{1}$ Chercheur qualifié du Fonds National de la Recherche Scientifique, Bruxelles. Postal address: Physique Théorique, CP 225, ULB, Bd du Triomphe, B-1050 Brussels, Belgium.

* Work supported in part by NATO under contract $0656 / 84$. 
Here $\Delta m^{2}=\left|m_{\nu_{c}}^{2}-m_{\nu_{a}}^{2}\right|$ and $\nu_{a}$ could either be $\nu_{\mu}$ or $\nu_{\tau}$ or some other exotic neutral lepton ${ }^{\star}$. Suppose the conversion takes place between $\nu_{\mathrm{e}}$ and $\nu_{\mu}$, eq. (1) implies that either $m_{\nu_{\mathrm{e}}}$ and $m_{\nu_{\mu}}$ must be extremely degenerate or

$$
m_{\nu_{\mathrm{e}}}, m_{\nu_{\mu}} \leq 10^{-2} \mathrm{eV} .
$$

We will assume eq. (3) to hold (although there is no fundamental reason to exclude the other possibility). We will not focus on how to generate such a small neutrino mass. Many suggestions have, in fact, already been given in the literature. Instead, if the solar neutrino puzzle is indeed confirmed and the mass of the neutrino is constrained by eq. (1), we wonder whether it is possible to have sizable $C P$ violation effects in the lepton sector. As far as we can determine, this is still an open question.

In the standard $\mathrm{SU}(2)_{\mathrm{L}} \times \mathrm{U}(1)_{\mathrm{Y}}$ model where the smallness of the neutrino mass reaches its limit $m_{v_{e, \mu, \tau}}=0$, although $C P$ violation occurs in the quark sector via the Kobayashi-Maskawa (KM) mechanism [5], $C P$ violation in the lepton sector appears to be vanishingly small ${ }^{\star \star}$. There is not any experimental evidence so far to support the idea that $C P$ is not a good symmetry of the leptons. In fact, in attempting to find $C P$ violation signatures in the lepton sector, measurements have, for instance, set significant limits [6] on the electron electric dipole moment (denoted by $d_{\mathrm{e}}$ hereafter)

$$
d_{\mathrm{e}} \leq 10^{-24} \mathrm{e} \cdot \mathrm{cm} \text {. }
$$

On the other hand, theories beyond the standard model always seem to favour the introduction of $C P$ violation. In view of the prospective experiments that may greatly help us to understand the mystery of $C P$ violation, it becomes interesting to explore the possibility of violating $C P$ symmetry in the lepton sector with the mass of the neutrinos being extremely small. We show that in extended theories it is possible to have a sizable effect even if the masses of the neutrinos are zero. In particular, in the model we discuss we find that $d_{\mathrm{e}}$ could naturally be of the order of the present experimental bound eq. (4) and the muon longitudinal polarization $P_{\mathrm{L}}$ in the decay $\mathrm{K}_{\mathrm{L}} \rightarrow \bar{\mu} \mu$ could well be within the range accessible to future experiments.

The paper is organized as follows. In sect. 2 we present a neutrino mass matrix, and discuss the possibility of evading potential constraints from the neutrino mass on $C P$ violation observables in the lepton sector. Within our framework, we then examine $d_{\mathrm{e}}$ and $P_{\mathrm{L}}$ in sect. 3. We show that even in the limit where the mass of the

* Eqs. (1) and (2) were derived for left-handed neutrino oscillation, in which case neutral-current contributions cancel exactly; neutral-current contributions (although they do not cancel in the case of $\nu_{\mathrm{e}}$ and an exotic neutral lepton conversion) are relatively small and the above numbers will also be a good approximation for this case.

$\star \star$ A vanishingly small electric dipole moment of the electron can be induced in the KM model through the electric dipole moment of $W$, which can be generated from quark loops. 
light neutrino vanishes these effects can be very large. Phenomenological restrictions on the parameters of our mass matrix are analyzed in sect. 4. A realization of our mass matrix through spontaneous symmetry breaking is illustrated in the appendix.

\section{A neutrino mass matrix}

In order to illustrate the idea, it is convenient to begin with some considerations on a neutrino mass matrix without reference to its origin. For every lepton generation we assume that there are three neutral Weyl spinors

$$
\nu_{\mathrm{L}}, \quad \nu_{\mathrm{R}}, S_{\mathrm{L}} .
$$

Here $\mathrm{L}, \mathrm{R}$ are the usual chiral indices. Once we consider an explicit realistic model, two of these fields, say $\nu_{L, R}$, will be identified as the "ordinary" neutrinos, which may appear for example in left-right [7] or $\mathrm{SO}(10)$ models. The other field, $S_{\mathrm{L}}$, will be considered as an exotic species which is a singlet under the gauge group. This extra fermion arises in some models inspired by the superstring, where often one is led to a mass matrix (in the basis $\left.\nu_{\mathrm{L}},\left(\nu_{\mathrm{R}}\right)^{\mathrm{c}}, S_{\mathrm{L}}\right)$ of the form [8]

$$
\boldsymbol{M}_{\nu}=\left(\begin{array}{ccc}
0 & m & 0 \\
m^{\mathrm{T}} & 0 & M^{\mathrm{T}} \\
0 & M & m_{\sigma}
\end{array}\right)
$$

Here $m, M$ and $m_{\sigma}$ are $n \times n$ matrices with $n$ being the number of generations. Such a neutrino mass matrix has been suggested [9] recently by Gonzalez-Garcia and Valle to study neutrino-Majoron decays within the context of an $\mathrm{SU}(2)_{\mathrm{L}} \times \mathrm{U}(1)_{\mathrm{Y}}$ gauge theory.

In the limit $m_{\sigma} \rightarrow 0$, if the mixing between different generations is negligible, then for each generation a specific lepton number is conserved, and the interactions can be rewritten in terms of a massless left-handed neutrino and a massive Dirac neutrino. The unitary matrix which diagonalizes eq. (6) according to

$$
V M_{\nu} V^{\mathrm{T}}
$$

is then, for each generation of fermions,

$$
\boldsymbol{V}=\left(\begin{array}{ccc}
\frac{M}{\sqrt{M^{2}+m^{2}}} & 0 & -\frac{m}{\sqrt{M^{2}+m^{2}}} \\
\frac{i m}{\sqrt{2\left(M^{2}+m^{2}\right)}} & -\frac{i}{\sqrt{2}} & \frac{i M}{\sqrt{2\left(M^{2}+m^{2}\right)}} \\
\frac{m}{\sqrt{2\left(M^{2}+m^{2}\right)}} & \frac{1}{\sqrt{2}} & \frac{M}{\sqrt{2\left(M^{2}+m^{2}\right)}}
\end{array}\right) .
$$


The phase $i$ in eq. (8) is added to make the neutrino mass positive. It then follows from eqs. (7) and (8) that the mass eigenstate neutrinos are related to their weak eigenstates via the relation

$$
\left(\begin{array}{l}
\nu_{1} \\
\nu_{2} \\
\nu_{3}
\end{array}\right)=V\left(\begin{array}{c}
\nu_{\mathrm{L}} \\
\left(\nu_{\mathrm{R}}\right)^{c} \\
S_{\mathrm{L}}
\end{array}\right) .
$$

Notice that $\nu_{\mathrm{R}}$ does not contribute to $\nu_{1}$. The masses of the neutrinos are given by

$$
m_{v_{1}}=0, \quad m_{\nu_{2,3}}=\sqrt{M^{2}+m^{2}} .
$$

Since $\nu_{2}$ and $\nu_{3}$ are degenerate in mass, we can combine them to form a four component Dirac spinor. The above mechanism of generating massless neutrinos was first suggested by Wolfenstein and Wyler [10]. A realization of such a scheme was discussed [10] in $\mathrm{SO}(10)$ models.

Now, we add to eq. (8) the mass term $m_{\sigma}$. Once this term is added no zero eigenvalue subsists in general, so neutrinos must then be regarded as Majorana particles with no conserved lepton number. Of special interest, we consider

$$
m_{\sigma}, m \ll M
$$

In this case, one finds

$$
m_{\nu_{1}} \approx(m / M)^{2} m_{\sigma}, \quad m_{\nu_{2,3}} \approx M \pm m_{\sigma} / 2,
$$

and the unitary matrix $U$ becomes

$$
\boldsymbol{U}=\boldsymbol{V}+\mathrm{O}\left(m m_{\boldsymbol{a}} / M^{2}\right) .
$$

In $S U(2)_{\mathrm{L}} \times \mathrm{U}(1)_{\mathrm{Y}}$ models (with the exception of mirror models [11]), both $\nu_{\mathrm{R}}$ and $S_{\mathrm{L}}$ may have to be gauge singlets and, therefore, are sterile with respect to the gauge interactions. As a result, the only connection between $\nu_{\mathrm{L}}$ and $\nu_{\mathrm{R}}$ or $S_{\mathrm{L}}$ is via scalar interactions which are determined by the Yukawa coupling and the Higgs potential of the model. Such interactions are normally weaker than the gauge interactions because of the smallness of the "ordinary" lepton mass. Therefore, $C P$ violation through scalar interactions is likely to remain small. This possibility has been reviewed recently by Langacker and London [12]. Of course, with a complicated Higgs structure it is also possible to introduce a sufficiently large amount of $C P$ violation. For simplicity, we will not discuss this possibility any further in the present paper.

On the other hand, one attractive extension of the standard electroweak model uses $\mathrm{SU}(2)_{\mathrm{L}} \times \mathrm{SU}(2)_{\mathrm{R}} \times \mathrm{U}(1)$ as a gauge group [7]. In these theories we can identify, 
say $\nu_{R}$, as the neutral member of the $\mathrm{SU}(2)_{\mathrm{R}}$ lepton doublet, and hence $\nu_{\mathrm{R}}$ will participate in weak interactions through right-handed currents (i.e. a virtual $W_{R}$ exchange). If the mass of $\mathrm{W}_{\mathrm{R}}, M_{\mathrm{W}_{\mathrm{R}}}$, is not too heavy, the effect of this interaction will be very much sizable ${ }^{\star}$. We point out that in eq. (6), there is no term $\bar{\nu}_{\mathrm{L}} S_{\mathrm{R}}$ corresponding to the entry $\bar{\nu}_{\mathrm{R}} S_{\mathrm{L}}$. Consequently, the mass matrix, although consistent with spontaneous breaking of the $\mathrm{SU}(2)_{\mathrm{L}} \times \mathrm{SU}(2)_{\mathrm{R}} \times \mathrm{U}(1)$ gauge symmetry, does not explicitly exhibit the usual left-right discrete symmetry. (In fact, we have explicitly treated $S_{\mathrm{L}}$ as a two-components left-handed spinor.) Before going into the details, we would like to outline the differences between the present situation and the more usual LR models. For some $C P$-violating observables, like $d_{\mathrm{e}}$ and $P_{\mathrm{L}}$, the effect is in general proportional to a factor

$$
m\left(M_{\mathrm{W}_{\mathrm{L}}} / M_{\mathrm{W}_{\mathrm{R}}}\right)^{2} .
$$

In the simplest left-right models, where the neutrino mass matrix takes the form

$$
\left(\begin{array}{cc}
0 & m \\
m^{\mathrm{T}} & M
\end{array}\right)
$$

and thus the light neutrino mass is given by the usual "see-saw" formula [14]

$$
m_{1} \approx m^{2} / M
$$

the factor in eq. (14) becomes

$$
\sqrt{m_{1} M}\left(M_{\mathrm{W}_{\mathrm{L}}} / M_{\mathrm{W}_{\mathrm{R}}}\right)^{2} .
$$

Since $M \sim M_{\mathrm{W}_{\mathrm{R}}}$, which has a well established lower bound $\left(M_{\mathrm{W}_{\mathrm{R}}}>1.6 \mathrm{TeV}\right)$, a sufficiently small neutrino mass will therefore render it undetectable. In particular, in the limit $m_{1} \rightarrow 0$, the effect under consideration vanishes. This happens because in this simple scheme the only way to make the neutrino massless is to require $m=0$.

The situation changes drastically if we consider the mass matrix given by eq. (6). Now, the light neutrino mass is given by the modified see-saw formula eq. (12). We see that as long as $m_{\sigma}<M$, the light neutrino mass will be much more suppressed than that given by the ordinary see-saw formula because $m_{\nu_{1}}=\left(m_{\sigma} / M\right)\left(m^{2} / M\right)$. Thus, to have a small neutrino mass, we are no longer obligated to choose a very small $m$. In particular, in order to make $m_{\nu_{1}}=0$, we may just choose $m_{\sigma}$ rather than $m$ to be zero. Thus, in our extended model the factor which is relevant for the $C P$ violation (eq. (14)) is no longer directly related to the light neutrino mass. As a result, a large amount of $C P$ violation becomes possible.

\footnotetext{
* For a recent review of $C P$ violation in left-right models we refer to ref. [13].
} 
An alternative look at the mass matrix (for $m_{\sigma}=0$ ) uses $\nu_{ \pm}=\left(\nu_{\mathrm{R}}^{\mathrm{c}} \pm S_{\mathrm{L}}\right) / \sqrt{2}$, in the basis $\left(\nu_{\mathrm{L}}, \nu_{+}, \nu_{-}\right)$the mass matrix then reads:

$$
\boldsymbol{M}_{\nu}=\left(\begin{array}{ccc}
0 & m / \sqrt{2} & m / \sqrt{2} \\
m / \sqrt{2} & M & 0 \\
m / \sqrt{2} & 0 & -M
\end{array}\right) .
$$

In this "Majorana" basis, we get a different picture of what is happening. Both heavy Majorana neutrinos, with opposite $C P$ parities mix with $\nu_{\mathrm{L}}$ and should thus contribute a term of order $\mathrm{m}^{2} / M$ to its mass; the two contributions however cancel exactly, which allows to keep $m / M$ large. Because only one linear combination of the heavy neutrinos couples to the $\mathrm{W}_{\mathrm{R}}$ however, no such cancellation happens in diagrams involving these particles as intermediary virtual states. This leads to large $C P$ effects. The introduction of the lepton-number violating $m_{\sigma}$ introduces a perturbation to the above scheme, and the light neutrino acquires a small Majorana mass while the large mixing with $\nu_{\mathrm{R}}$ is maintained. Constraints on $m / M$ will be given in subsect. 3.1.

\section{Some interesting $C P$ violation effects}

We first examine the limiting case $m_{\sigma}=0$ and further assume, for simplicity, that the generation mixings of the leptons are negligible. For each generation, there is a $C P$ violation phase in the lepton sector if the left-right mixing, $\xi$, is not zero. This phase can be parametrized in terms of a complex $m_{a}$ ( $a$ is the generation index) with $m_{a}=D_{a} \mathrm{e}^{i \delta_{a}}$ and $D_{a}$ real. It is a trivial task to show that the rest of the phases can be rotated away by a simultaneous transformation of the fermion and gauge boson fields. In this phase convention, the factor $m_{a}^{2}$ in eq. (8) should be read as $D_{a}^{2}$. It then follows that for each generation, the charged current of the model can be written as

$$
L^{\mathrm{cc}}=-\frac{g_{\mathrm{L}}}{\sqrt{2}} \mathrm{~W}_{\mathrm{L}}^{\mu} \bar{l}_{\mathrm{L} a} \gamma_{\mu} \nu_{\mathrm{L} a}-\frac{g_{\mathrm{R}}}{\sqrt{2}} \mathrm{~W}_{\mathrm{R}}^{\mu} \bar{l}_{\mathrm{R} a} \gamma_{\mu} \nu_{\mathrm{R} a}+M_{\mathrm{W}_{\mathrm{R}}}^{2} \xi \mathrm{W}_{\mathrm{L}}^{\dagger} \mathrm{W}_{\mathrm{R}}+\text { h.c. }
$$

Substituting eqs. (8) and (9) into eq. (18), $L^{\mathrm{cc}}$ can equally be expressed in terms of the mass eigenstate neutrinos $\nu_{1,2,3}$.

\subsection{THE ELECTRIC DIPOLE MOMENT OF THE ELECTRON}

It is well known that a nonvanishing electric dipole moment (EDM) of a particle violates $C P$. Theoretical estimates for the EDM of the neutron have been made in many models [15]. A special consideration in the case of the neutron EDM is that a non-zero value can occur directly from weak interaction effects as well as from the 


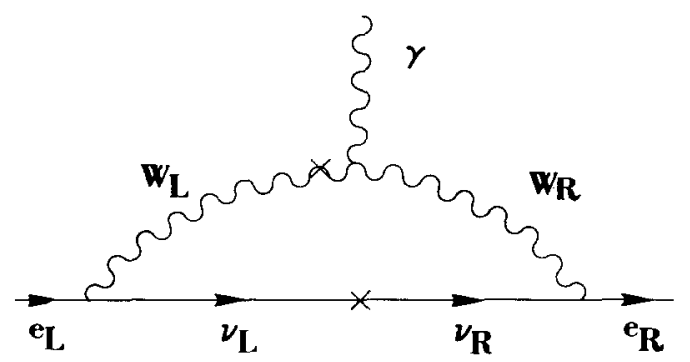

Fig. 1. One loop contributions to the electron electric dipole moment $d_{\mathrm{e}}$. The cross on the gauge boson line represents the left-right mixing. The cross on the internal neutrino line represents a Dirac mass term insertion.

QCD $\theta$ parameter. The EDM of the electron [16] is, however, a pure weak effect. The value of the EDM of the electron, $d_{\mathrm{e}}$, is vanishingly small in the KM model. In $\mathrm{SU}(2)_{\mathrm{L}} \times \mathrm{SU}(2)_{\mathrm{R}} \times \mathrm{U}(1)$ left-right models, allowing $m_{v_{\mathrm{e}}}$ to be of the order of a few $\mathrm{eV}$, previous estimates found [17]

$$
d_{e} \leqslant 10^{-26} e \cdot \mathrm{cm}
$$

which is not far from the present experimental limit (4). On the other hand, if one assumes that the mass of the neutrino is zero, then $d_{\mathrm{e}}$ vanishes in these models.

The induced effective EDM interaction of the electron, $i d_{\mathrm{e}} \bar{e} \sigma_{\mu \nu} q^{\mu} \gamma_{5} e \epsilon^{\nu}$, is generated by the Feynman graph depicted in fig. 1. The result of the computation of this graph is

$$
d_{\mathrm{e}} \approx\left(\sqrt{2} G_{\mathrm{F}} / 4 \pi^{2}\right) e_{1} D_{1} \xi \sin \delta_{1} f\left(M_{1}^{2} / M_{\mathrm{W}_{\mathrm{R}}}^{2}\right)
$$

Here we have used $M_{\mathrm{W}_{\mathrm{R}}} \gg M_{\mathrm{W}_{\mathrm{L}}}$ and $g_{\mathrm{L}}=g_{\mathrm{R}} \cdot f$ is a smooth function of its argument varying from a value of 1 for $M_{1} \ll M_{\mathrm{W}_{\mathrm{R}}}$ to a value of $1 / 4$ for $M_{1} \gg M_{\mathrm{W}_{\mathrm{R}}}$ ( $M_{1}$ is the first generation heavy neutrino mass.) Thus, for reasonable choice of the parameters, $f \sim 1$.

Stringent upper bounds on $\xi$ have been established [18], one finds (for a short review see the first item of ref. [18])

$$
\xi \leq 5 \times 10^{-3}
$$

Under this constraint, eq. (20) leads to $d_{\mathrm{e}} \leqslant 10^{-26} e \cdot \mathrm{cm}$ for $D_{1}-1 \mathrm{MeV}$. As we mentioned before, the choice of $D_{1}$ in the simple left-right models is restricted by the experimental limit on $\nu_{\mathrm{e}}$ and the see-saw relation (16). In our case, the situation becomes completely different. Since the mass of the neutrino $\nu_{1}$ is always zero (see eq. (10)), the constraints on the value of $D_{1}$ are only minimal. 
The key reason is that the massless neutrino is a mixture of $S_{\mathrm{L}}$ and $\nu_{\mathrm{L}}$ only; $\nu_{\mathrm{R}}^{\mathrm{c}}$ does not appear, as this would violate lepton number. Therefore, no polarization effects are detectable in $\mu$ or semileptonic decays, which makes the detection of the mixing difficult [19]. The main effect is to suppress all currents involving neutrinos by a factor $\cos \phi$ where $\nu_{1}=\nu_{\mathrm{L}} \cos \phi-S_{\mathrm{L}} \sin \phi$. Assuming for simplicity similar mixing angles for all generations, we see that $\mu$-e universality is not affected, that the amplitude of $\mu$ decay is affected by $\cos ^{2} \phi$, while semileptonic charged currents receive a factor $\cos \phi$ times the usual Kobayashi-Maskawa matrix elements. Neutral current effects are affected by $\cos ^{2} \phi$. Since the determination of the KM matrix elements is based upon the analysis of $\mu$ decay, we cannot use them here. The most prominent signal comes from a comparison of neutral versus charged currents in neutrino scattering [20] (or a comparison between the $\mathrm{W}_{\mathrm{L}}$ mass and the standard expectation, if we were in the standard model). Even a $1 \%$ effect in the ratio of the cross sections allows for $|\sin \phi|<0.10$. From there one finds that a mixing between $\nu_{\mathrm{L}}$ and $S_{\mathrm{L}}$, which is approximately given by $D_{1} / M_{1}$, of $10 \%$ is allowed. As a result, in our model $d_{\mathrm{e}}$ can be as large as the present experimental limit provided we choose $D_{1} \sim 100 \mathrm{MeV}, \xi \sim 10^{-3}, \sin \delta_{1} \sim 1$ and $M_{\mathrm{W}_{\mathrm{R}}} \sim 1 \mathrm{TeV}$.

\subsection{MUON LONGITUDINAL POLARIZATION IN THE DECAY $\mathrm{K}_{\mathrm{L}} \rightarrow \bar{\mu} \mu$}

One of the interesting features of our model is that it produces relatively large values for some $C P$ violation observables. $C P$ violation can show up in places where the other $C P$-violating models do not expect to have any appreciable contributions. The muon longitudinal polarization $P_{\mathrm{L}}$ in the decay $\mathrm{K}_{\mathrm{L}} \rightarrow \bar{\mu} \mu$ is of special interest. $P_{\mathrm{L}}$ is defined as

$$
P_{\mathrm{L}}=\frac{N_{\mathrm{L}}-N_{\mathrm{R}}}{N_{\mathrm{L}}+N_{\mathrm{R}}}
$$

where $N_{\mathrm{L}, \mathrm{R}}$ denote the number of left- and right-handed muons. It has been noted long ago that the observation of $P_{\mathrm{L}}$ in this decay would constitute evidence for $C P$ violation [21]. Furthermore, a detection of $P_{\mathrm{L}}>10^{-3}$ would imply a violation of $C P$ symmetry beyond the well known $\mathrm{K}_{1}-\mathrm{K}_{2}$ mixing, $\epsilon=2.3 \times 10^{-3}$.

To calculate $P_{\mathrm{L}}$ we follow Herczeg [22] and write the effective Hamiltonian as

$$
H_{\mathrm{eff}}=\frac{G_{\mathrm{F}}}{\sqrt{2}}\left(\bar{s} \gamma_{5} d\right)\left(a \bar{\mu} \gamma_{5} \mu+i b \bar{\mu} \mu\right)+\text { h.c. }
$$

In general $a$ is complex because it includes the standard absorptive part of the two-photon intermediate state whereas the $C P$-violating part $b$ is real. Using the known value of $\operatorname{Im} a$ and the experimental rate $\left(\Gamma\left(\mathrm{K}_{\mathrm{L}} \rightarrow \bar{\mu} \mu\right) \approx 1.16 \times 10^{-6} \mathrm{eV}\right)$ one finds

$$
P_{\mathrm{L}}=1.8 \times b
$$




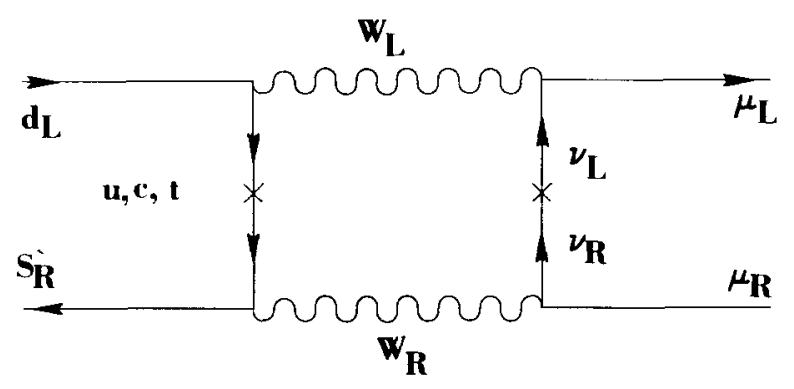

Fig. 2. One loop contributions to $P_{\mathrm{L}}$ in the decay $\mathrm{K}_{\mathrm{L}} \rightarrow \bar{\mu} \mu$.

Calculations for $b$ and thus $P_{\mathrm{L}}$ have been carried out in various gauge models [23]. This contribution adds up to the one originating from $\mathrm{K}_{1}-\mathrm{K}_{2}$ mixing, the latter being of the order $\epsilon$. In most cases the new contribution to $P_{\mathrm{L}}$ is found to be less than $10^{-3}$. In $\mathrm{SU}(2)_{\mathrm{L}} \times \mathrm{SU}(2)_{\mathrm{R}} \times \mathrm{U}(1)$ models, neglecting scalar interactions, the dominant contribution to the parameter $b$ is found arising from the left-right box diagram (fig. 2). Neglecting the external masses, one finds

$$
b \approx \frac{G_{\mathrm{F}}}{2^{3 / 2} \pi^{2}} \sin \theta_{C} \sin \delta_{2} m_{\mathrm{c}} D_{2}\left(\frac{M_{\mathrm{W}_{\mathrm{L}}}^{2}}{M_{\mathrm{W}_{\mathrm{R}}}^{2}}\right)^{2}\left(\frac{M_{\mathrm{W}_{\mathrm{R}}}}{M_{2}}\right)^{2} \ln \frac{M_{2}^{2}}{M_{\mathrm{W}_{\mathrm{R}}}^{2}},
$$

where $m_{\mathrm{c}}$ is the c-quark mass and $\theta_{\mathrm{C}}$ is the Cabibbo angle. In evaluating the diagram we have used, again for simplicity, $M_{2} \gtrsim M_{\mathrm{W}_{\mathrm{R}}}$ and $g_{\mathrm{L}}=g_{\mathrm{R}}$. We have ignored the $C P$ violation phases of the quark sector, which are not expected to affect qualitatively the result if the leptonic phases are large.

Previous investigations found that in the simple left-right models $b$ cannot be sufficiently large because our choice on $D_{2}$ is constrained by $m_{v_{\mu}} \sim D_{2}^{2} / M_{\mathrm{W}_{\mathrm{R}}} \leqslant$ $0.25 \mathrm{MeV}$. There, for a reasonable choice of the parameters one finds $P_{\mathrm{L}} \sim 10^{-4}$. In the present case, such a constraint no longer exists. Assuming $M_{2} \sim M_{\mathrm{W}_{\mathrm{R}}} \sim 1 \mathrm{TeV}$ (we can in fact take $D_{2} / M_{2}$ as large as $10 \%$, the limit considered above) we get $D_{2} \lesssim 100 \mathrm{GeV}$. As a result, we have from eqs. (24) and (25)

$$
P_{\mathrm{L}} \leqslant 0.5 \times 10^{-1} \text {. }
$$

We should also remark that choosing $D_{2}$ larger than $M_{\mathrm{W}_{\mathrm{R}}}$ is not allowed because the model simply gives too large a value for the decay rate. In any case, we expect that in the present model $P_{\mathrm{L}}$ could be within the range $10^{-1}-10^{-3}$.

A priori, allowing different $\nu_{\mathrm{L}}-S_{\mathrm{L}}$ mixings for e and $\mu$ would create problems with $\mathrm{e}-\mu$ universality. The $1 \%$ effect corresponding to (26) seems however compatible with experimental data [24]. Adding a sufficiently small $m_{\sigma}$ to the neutrino mass 
matrix (6) will not change qualitatively the results discussed above. A realization of such a mass matrix will be discussed in the appendix.

\section{Constraints on the neutrino mass matrix}

Evidently, the addition of $m_{\sigma}$ breaks the lepton number, and would induce some lepton number violating processes like the neutrinoless double $\beta$ decay. Also, allowing for some amount of mixing between the different generations so that the MSW mechanism is operative (see eq. (2)) will result in a substantial decay $\mu \rightarrow$ e $\gamma$ even in the limit $m_{\sigma} \rightarrow 0$. It is therefore important to examine these effects to see if additional constraints can be obtained.

\subsection{NEUTRINOLESS DOUBLE $\beta$ DECAY}

It is straightforward to show [25] that the dominant term to the neutrinoless double $\beta$ decay is the usual $m_{\nu_{\mathrm{e}}}$ term which is left-left. Such a contribution is negligibly small once we require $m_{\nu_{\mathrm{c}}} \leqslant 10^{-2} \mathrm{eV}$. Also, it is easy to show that contributions from left-right mixing are completely negligible. As a result, considerations on the neutrinoless double $\beta$ decay do not provide any useful constraint on our model.

\subsection{THE DECAY $\mu \rightarrow \mathrm{e} \gamma$}

The dominant contribution to this process is generated by the graph in fig. 3. To a good approximation $\left(M \sim M_{\mathrm{W}_{\mathrm{R}}} \gg M_{\mathrm{W}_{\mathrm{L}}}, D_{2} \gg D_{1}\right)$ we find that the transition amplitude of the decay is

$$
T(\mu \rightarrow \mathrm{e} \gamma) \approx\left(e G_{\mathrm{F}} / 8 \pi^{2}\right) D_{2} \xi \sin \theta
$$

where $\theta$ is the generation mixing. This yields a branching ratio for the decay

$$
\mathrm{BR}(\mu \rightarrow \mathrm{e} \gamma)=\frac{3 \alpha}{4 \pi}\left(\frac{D_{2}}{m_{\mu}}\right)^{2} \xi^{2} \sin ^{2} \theta
$$

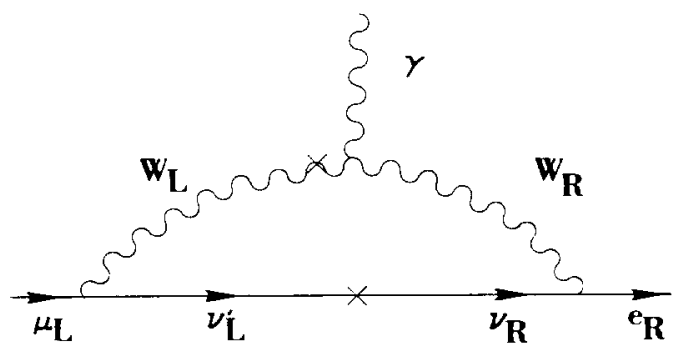

Fig. 3. One loop contributions to the decay $\mu \rightarrow \mathrm{e} \gamma$. 
In eq. (28), $\alpha=\mathrm{e}^{2} / 4 \pi^{2}$. The present experimental limit requires $\mathrm{BR}<10^{-10}$. If $\sin \theta$ is negligible, eq. (28) practically does not provide any additional constraints on the parameters of the model.

However, if we assume $\theta$ to be within the range given by eq. (2) so that the MSW mechanism is operative, we find from eq. (28)

$$
D_{1} \leq m_{\mu} .
$$

An important consequence of this constraint is (eq. (25))

$$
P_{\mathrm{L}} \leq 0.5 \times 10^{-4} \text {. }
$$

This is the typical value of $P_{\mathrm{L}}$ of the simple left-right model discussed in ref. [22]. Notice that this result depends on the choice of the generation mixing not the value of $m_{\sigma}$. Therefore, even in the limit $m_{\sigma}=0, D_{2}$ and thus $P_{\mathrm{L}}$ have to be bounded by eqs. (29) and (30) respectively as long as $\sin ^{2} \theta \geq 10^{-3}$.

\subsection{COSMOLOGICAL CONSTRAINT}

Since (1) the heavy neutrinos (the $\nu_{2}$ 's and the $\nu_{3}$ 's) have a sufficiently large mass $\left(\sim M_{\mathrm{W}_{\mathrm{R}}}\right)$, and (2) they are not strictly sterile (they interact via right-handed currents), the stability of these particles will not disturb our present understanding of the universe. The mass of the lighter neutrinos is given by the modified see-saw formula (12). If these particles are stable, they must be lighter than about $50 \mathrm{eV}$. This amounts to requiring all $m$ 's and $M$ 's and $m_{\sigma}$ 's to be such

$$
\left(\frac{m}{M}\right)^{2} m_{\sigma} \leq 50 \mathrm{eV}
$$

It is easy to see that such a constraint is automatically satisfied if we require the neutrinos masses to be in the range where the MSW mechanism is operative. Therefore, cosmological considerations will not provide any additional constraint on our model.

\section{Summary}

We have explored the possibility of introducing large $C P$ violation effects in the lepton sector while retaining small or vanishing neutrino masses. We have shown that with a suitable extension of the neutral lepton content, $C P$ violation can occur in the lepton sector even with massless neutrinos. In the $\mathrm{SU}(2)_{\mathrm{L}} \times \mathrm{SU}(2)_{\mathrm{R}} \times \mathrm{U}(1)$ gauge model we have considered, we find that the electron electric dipole moment can be as large as the present experimental limit. Moreover, if the mixing between different generations is negligible, the muon longitudinal polarization in the decay 
$\mathrm{K}_{\mathrm{L}} \rightarrow \bar{\mu} \mu$ can be within the range $10^{-1}-10^{-3}$ and is therefore accessible to currently planned experiments. On the other hand, allowing the neutrinos to have a very small mass and a sizable mixing so that the MSW mechanism is operative, we find that $d_{\mathrm{e}}$ can still be large while $P_{\mathrm{L}}$ has to be smaller than about $10^{-4}$.

This work was supported in part by the US Department of Energy.

\section{Appendix A}

In this appendix we present an explicit model to illustrate how our mass matrix can be realized within the framework of $\mathrm{SU}(2)_{\mathrm{L}} \times \mathrm{SU}(2)_{\mathrm{R}} \times \mathrm{U}(1)$ gauge theories. The fermions of the model consist of those in the standard $\mathrm{SU}(2)_{\mathrm{L}} \times \mathrm{U}(1)_{\mathrm{Y}}$ model plus two additional neutrinos $\nu_{\mathrm{R}}, S_{\mathrm{L}}$. More specifically, the lepton content of the model is given by

$$
\psi_{\mathrm{L} a}\left(\frac{1}{2}, 0,-\frac{1}{2}\right)=\left(\begin{array}{c}
\nu_{\mathrm{L} a} \\
l_{\mathrm{L} a}
\end{array}\right), \quad \mu_{\mathrm{R} a}\left(0, \frac{1}{2},-\frac{1}{2}\right)=\left(\begin{array}{c}
\nu_{\mathrm{R} a} \\
l_{\mathrm{R} a}
\end{array}\right), \quad S_{\mathrm{L} a}(0,0,0) .
$$

Here $a=1,2, \ldots, n$ is the family index. We have introduced leptons in an asymmetrical way (because there is no $S_{\mathrm{R}}$ in our model). As a result, our model does not explicitly exhibit the usual left-right discrete symmetry. (Introducing fermions in an asymmetrical way is of course not new, in fact, this happens in the standard electroweak model.) The Higgs sector consists of five multiplets

$$
\Phi_{1,2}\left(\frac{1}{2}, \frac{1}{2}, 0\right), \quad H_{\mathrm{L}}\left(\frac{1}{2}, 0, \frac{1}{2}\right), \quad H_{\mathrm{R}}\left(0, \frac{1}{2}, \frac{1}{2}\right), \quad \sigma(0,0,0) .
$$

The Yukawa couplings of the leptons are taken to be (for simplicity, we suppress the family index)

$$
\bar{\phi}_{\mathrm{L}}\left(f \Phi_{1}+f^{\prime} \Phi_{1}^{\mathrm{c}}\right) \psi_{\mathrm{R}}+F \bar{\psi}_{\mathrm{R}} H_{\mathrm{R}} S_{\mathrm{L}}+h \sigma \bar{S}_{\mathrm{L}}^{\mathrm{c}} S_{\mathrm{L}}+\text { h.c. },
$$

where $\Phi^{\mathrm{c}}=\sigma_{2} \Phi^{*} \sigma_{2}$ and $\sigma_{1,2,3}$ are the Pauli matrices. The assumption of only four terms in eq. (A.3) among the six fields requires two U(1) symmetries. Evidently, one of them is the gauged $\mathrm{U}(1)$ symmetry, and the other is a global $\mathrm{U}(1)$ which could be taken as the usual lepton number with the following assignments

$$
\psi_{\mathrm{L}, \mathrm{R}}, S_{\mathrm{L}}:(+1) ; \quad \sigma:(-2) .
$$

We can restrict this global U(1) symmetry to a discrete symmetry by requiring that the singlet $\sigma$ belongs to a real representation of the gauge group. The consequences of introducing a real scalar singlet has also been discussed in the literature [26]. In this case, the discrete symmetry can be taken as

$$
\left(l_{\mathrm{L}, \mathrm{R}}, S_{\mathrm{L}}\right) \rightarrow i\left(l_{\mathrm{L}, \mathrm{R}}, S_{\mathrm{L}}\right), \quad \sigma \rightarrow-\sigma .
$$


Under this symmetry the rest of the fields remain the same but we require

$$
\Phi_{2} \rightarrow-\Phi_{2} .
$$

Once the scalars develop vacuum expectation values

$$
\left\langle\Phi_{1,2}\right\rangle=\left(\begin{array}{cc}
k_{1,2} & 0 \\
0 & k_{1,2}^{\prime}
\end{array}\right), \quad\left\langle H_{\mathrm{L}}\right\rangle=\left(\begin{array}{c}
0 \\
\nu_{\mathrm{L}}
\end{array}\right), \quad\left\langle H_{\mathrm{R}}\right\rangle=\left(\begin{array}{c}
0 \\
V_{\mathrm{R}}
\end{array}\right), \quad\langle\boldsymbol{\sigma}\rangle=\boldsymbol{\sigma}^{\prime},
$$

the neutrino mass matrix arising from eq. (A.3) becomes exactly the one given by eq. (6) with

$$
m=f k_{1}+f^{\prime} k_{1}^{\prime}, \quad M=F V_{\mathrm{R}}, \quad m_{\sigma}=h \sigma^{\prime} .
$$

The hierarchy among the different terms (eq. (11)) arises from the Higgs potential which we briefly describe below.

The Higgs potential that contains only $\Phi_{1}$ and $H_{\mathrm{L}, \mathrm{R}}$ has been discussed before [27]. The result is well known. One can show that there is a large range of parameters in which the vacuum expectation values of the Higgs fields have the following hierarchical structure

$$
v_{\mathrm{L}} \sim \frac{\left(k_{1}+k_{1}^{\prime}\right)^{2}}{V_{\mathrm{R}}} \ll k_{1}, k_{1}^{\prime} \ll V_{\mathrm{R}} .
$$

The small value of $v_{\mathrm{L}}$ is induced by the trilinear coupling

$$
\mu H_{\mathrm{L}}^{\dagger} \Phi_{1} H_{\mathrm{R}}
$$

in the Higgs potential. Here it is assumed that $\mu$ is of the order $\left(k_{1}+k_{1}^{\prime}\right)$.

Including $\Phi_{2}$ and $\sigma$ will add additional terms that respect all the symmetries of the Higgs potential. The size of $\left\langle\Phi_{2}\right\rangle$ is constrained by the $\mathrm{SU}(2)_{\mathrm{L}}$ breaking so, naturally, we have

$$
\left\langle\Phi_{2}\right\rangle \sim\left\langle\Phi_{1}\right\rangle
$$

The singlet $\sigma$ will receive a large positive mass correction $V_{\mathrm{R}}^{2}$ due to the coupling $H_{\mathrm{R}}^{\dagger} H_{\mathrm{R}} \sigma^{2}$ in the Higgs potential. Therefore the vacuum expectation value of $\sigma$ arises only because of the term

$$
\sigma H_{\mathrm{L}}^{\dagger} \Phi_{2} H_{\mathrm{R}}
$$

This is the reason why in our model an additional $\Phi\left(\frac{1}{2}, \frac{1}{2}, 0\right)$ multiplet is needed. 
This induces

$$
\sigma^{\prime} \sim k v_{\mathrm{L}} / V_{\mathrm{R}} \sim k\left(k^{2} / V_{\mathrm{R}}^{2}\right)
$$

Here we have assumed, for simplicity, that the orders of magnitude of all the $k$ 's are the same. In the last step of (A.13) we have used (A.9). Also, for simplicity, we have taken all the dimensionless coupling constants in the Higgs potential to be of the order of unity.

To get a feeling for the order of magnitude of our results we take $k \sim 100 \mathrm{GeV}$ and $V_{\mathrm{R}} \sim$ a few $\mathrm{TeV}$ so that $M_{\mathrm{W}_{\mathrm{R}}} \sim 1 \mathrm{TeV}$. Take $m \sim m_{\mu} \approx 100 \mathrm{MeV}$ we find for $h \sim 10^{-1}$

$$
m_{1} \sim 10^{-2} \mathrm{eV}
$$

A much smaller neutrino mass can easily be obtained if we choose all the coupling constants of those terms that are responsible for the spontaneous breaking of our discrete symmetry to be very small. Tuning these parameters small is of course natural in the technical sense [28] because in the limit that these couplings equal zero, the model has an enlarged symmetry.

The zero entries in eq. (6) will receive radiative corrections in higher order of perturbation interactions. However, one can easily check that all these corrections are very small and therefore negligible. Finally, the limiting case $m_{o}=0$, which appears to be of most interest, simply corresponds to a model that does not contain the Higgs fields $\Phi_{2}$ and $\sigma$ but has a conserved lepton number (A.4).

\section{References}

[1] S.M. Bilenky and S.T. Petcov, Rev. Mod. Phys. 59 (1987) 671

[2] L. Wolfenstein, Phys. Rev. D17 (1978) 2369; D20 (1979) 2634; S.P. Mikheyev and A. Yu. Smirnov, JETP 91 (1986) 7

[3] R. Davis et al., Phys. Rev. Lett. 20 (1968) 1205;

J.K. Rowley, B.T. Cleveland and R. Davis, in Proc. Conf. on Solar neutrinos and neutrino astronomy, AIP Conf. Prof. No. 126 (AIP, New York, 1985) p. 1

[4] S.P. Rosen and J.M. Gelb, Phys. Rev. D34 (1986) 969

[5] M. Kobayashi and T. Maskawa, Prog. Theor. Phys. 49 (1973) 652

[6] T.G. Vold et al., Phys. Rev. Lett. 52 (1984) 2229;

V.V. Flambaum and I.B. Khriplovich, Zh. Eksp. Teor. Fiz. 89 (1985) 1505

[7] J.C. Pati and A. Salam, Phys. Rev. D10 (1974) 275;

R.N. Mohapatra and J.C. Pati, Phys. Rev. D11 (1975) 2558;

G. Senjanovic and R.N. Mohapatra, Phys. Rev. D12 (1975) 1502;

H. Fritzsch and P. Minkowski, Nucl. Phys. B103 (1976) 61

[8] R.N. Mohapatra and J.W.F. Valle, Phys. Rev. D34 (1986) 1642;

I. Antoniadis et al., Phys. Lett. B208 (1988) 209

[9] M.C. Gonzalez-Garcia and J.M.F. Valle, CERN-TH-5170 (1988)

[10] D. Wyler and L. Wolfenstein, Nucl. Phys. B218 (1983) 205

[11] J. Maalampi and M. Roos, University of Helsinki, HU-TFT-88-17

[12] P. Langacker and D. London, Phys. Rev. D38 (1988) 907

[13] R.N. Mohapatra, University of Maryland, UM PP \# 88-003 
[14] M. Gell-Mann, P. Ramond and R. Slansky, in Supergravity, ed. D Freedman and P. Van Nieuwenhuizen (North-Holland, Amsterdam, 1979);

T. Yanagida, in Proc. Workshop on the Baryon Number of the Universe and Unified Theories, Tsukuba, 1979, ed. O. Sawada and A. Sugamoto (National Lab for High Energy Physics, Tsukuba, Japan, 1979), p. 95;

R.N. Mohapatra and G. Senjanovic, Phys. Rev. Lett. 44 (1980) 912

[15] E.P. Shabalin, Sov. Phys. Usp. 26 (1983) 4;

G. Beall and A. Soni, Phys. Rev. Lett. 47 (1981) 552;

G. Beall and N.G. Deshpande, Phys. Lett. B132 (1983) 427;

G. Ecker, W. Grimus and H. Neufeld, Nucl. Phys. B229 (1983) 421;

M. Dugan et al., Nucl. Phys. B255 (1985) 413;

Ikaros I.Y. Bigi and A.I. Sanda, Phys. Rev. Lett. 58 (1987) 1604

[16] M. Fabbrichesi, P.M. Fishbane and R.E. Norton, Phys. Rev. D37 (1988) 1942;

S.M. Barr and A. Masiero, Phys. Rev. Lett. 58 (1987) 187;

Y. Kizukuri, Phys. Lett. B185 (1987) 183;

S.T. Petcov, Phys. Lett. B178 (1986) 57;

A. Zee, Phys. Rev. Lett. 55 (1985) 2382

[17] J.F. Nieves, D. Chang and P.B. Paul, Phys. Rev. D33 (1986) 3324;

J. Liu, Nucl. Phys. B271 (1986) 531

[18] J.-M. Frère, in Progress in Electroweak Interaction, ed. Tran Thanh Van (Editions Frontières 1986) p. 187 ;

I.I. Bigi and J.-M. Frère, Phys. Lett. B110 (1982) 255;

J. Donoghue and B. Holstein, Phys. Lett. B113 (1982) 382;

L. Wolfenstein, Phys. Rev. D29 (1984) 2130;

E. Masso, Phys. Rev. Lett. 52 (1984) 1956

[19] Miriam Leurer, University of Michigan, UM-TH-88-25 (1988);

P. Castoldi, J.-M. Frère, and G.L. Kane, Phys. Rev. D39 (1989) 2633

[20] J.-M. Frère, Nucl. Phys. B177 (1981) 389

[21] A. Pais and S.B. Treiman, Phys. Rev. 176 (1968) 1974;

L.M. Sehgal, Phys. Rev. 187 (1969) 2151

[22] P. Herczeg, Phys. Rev. D27 (1983) 1521

[23] D. Chang and R.N. Mohapatra, Phys. Rev. D30 (1984) 2005;

F.J. Botella and C.S. Lim, Phys. Rev. Lett. 56 (1986) 1651;

T. Kurimoto, Phys. Lett. B172 (1986) 357;

J. Liu, Phys. Rev. D36 (1987) 2178; Z. Phys. C37 (1987) 139;

J. Liu and L. Wolfenstein, Nucl. Phys. B289 (1987) 1

[24] D.I. Britton et al,, TRI-PP-88-52. (Presented at 3rd Conf. on Intersections between Particle and Nuclear Physics. Rockport, ME, May 1988.)

[25] M. Doi, T. Kotani and E. Takasugi, Prog. Theor. Phys. Suppl. 83 (1985) 1

[26] D. Chang, R.N. Mohapatra and M.K. Parida, Phys. Rev. Lett. 52 (1984) 1072

[27] G. Senjanovic, Nucl. Phys. B153 (1979) 334

[28] G. 't Hooft, in Recent developments in gauge theories, ed. G. 't Hooft et al. (Plenum Press, New York, 1980) 